\title{
HADWIGER'S TRANSVERSAL THEOREM IN HIGHER DIMENSIONS
}

\author{
JACOB E. GOODMAN AND RICHARD POLLACK
}

\section{INTRODUCTION}

In $1957 \mathrm{H}$. Hadwiger proved the following theorem [5], which generalized previous results independently obtained by B. Grünbaum [3] and V. Klee [7]:

Hadwiger's Transversal Theorem. Suppose $\mathscr{B}=\left\{B_{1}, \ldots, B_{n}\right\}$ is a family of pairwise disjoint compact convex sets in the real affine plane $\mathbf{A}^{2}$. Then the members of $\mathscr{B}$ have a common transversal line if and only if there is a linear ordering of $\mathscr{B}$ such that for every set of three indices $i, j, k$ there is a directed line which meets $B_{i}, B_{j}, B_{k}$ consistently with the ordering of $\mathscr{B}$.

Since then, attempts to prove corresponding results about hyperplane transversals in higher dimensions have met with little success; the only result in the same spirit as Hadwiger's which we are aware of and which holds in arbitrary dimension is the following theorem of F. A. Valentine [8], whose "special position" hypothesis is rather restrictive (we state it in dimension 3, as does Valentine, who then remarks that a corresponding result holds in higher dimensions):

Valentine's Theorem. Let $\mathscr{F}$ be a family of compact convex sets in $E_{3}$. Also suppose there exist three distinct planes $P_{i}(i=1,2,3)$ in $E_{3}$ containing a common line such that for each triple of members $M_{1}, M_{2}, M_{3}$ in $\mathscr{F}$ each pair of the triple is strictly separated from the remaining member of the triple by a translate of either $P_{1}$ or $P_{2}$ or $P_{3}$, and this correspondence is cyclic. If every four members of $\mathscr{F}$ have a common intersecting plane, then all the members of $\mathscr{F}$ have a common intersecting plane.

The purpose of this paper is to extend Hadwiger's theorem to hyperplane transversals of compact convex sets in $d$ dimensions, $d \geq 2$. The "ordering" of the sets is replaced by their "order type," and the condition that no two have

The main results of this paper were presented at the 90th Summer Meeting of the American Mathematical Society in Salt Lake City on August 5, 1987; received by the editors November 26, 1986.

1980 Mathematics Subject Classification (1985 Revision). Primary 52A20, 52A35; Secondary 05B35, $51 \mathrm{G} 05$.

The first author was supported in part by NSF Grant DMS-8501492 and PSC-CUNY Grant 666426.

The second author was supported in part by NSF Grant DMS-8501947. 
a common point by the condition that the family be "separated." (Our result is easily seen to include Valentine's theorem as a special case.)

Theorem 1. Suppose $\mathscr{B}=\left\{B_{1}, \ldots, B_{n}\right\}$ is a separated family of compact convex sets in real affine $d$-space $\mathbf{A}^{d}$. Then the members of $\mathscr{B}$ have a common transversal hyperplane if and only if there is a configuration of points $\mathscr{C}=\left\{x_{1}, \ldots, x_{n}\right\}$ in $\mathbf{A}^{d-1}$ such that for every set of $d+1$ indices $i_{1}, \ldots, i_{d+1}$ there is an oriented hyperplane $H$ which meets $B_{i_{1}}, \ldots, B_{i_{d+1}}$ consistently with the order type of $\mathscr{C}$.

A few definitions are in order. By a separated family of convex sets in $\mathbf{A}^{d}$ we mean one for which no $d$ members have a common transversal $d$-2-flat; e.g., separation in the plane just amounts to pairwise disjointness. (It is not hard to show that $\mathscr{B}$ is a separated family in $\mathbf{A}^{d}$ if and only if every set of $k$ elements of $\mathscr{B}$ can be separated from every set of $d-k$ other elements of $\mathscr{B}$ by a hyperplane; however we will never use this fact.) By the order type of a numbered set of points $\mathscr{C}=\left\{x_{1}, \ldots, x_{n}\right\}$ in $\mathbb{R}^{d}$ we mean the family of orientations of its $d+1$-tuples, i.e., the family

$$
\left(\operatorname{sgn} \operatorname{det}\left(\begin{array}{cccc}
1 & x_{i_{0}}^{1} & \cdots & x_{i_{0}}^{d} \\
\vdots & \vdots & \ddots & \vdots \\
1 & x_{i_{d}}^{1} & \cdots & x_{i_{d}}^{d}
\end{array}\right)\right)_{1 \leq i_{0}<\cdots<i_{d} \leq n}
$$

(see $\S 1$ of [4] for further details). Thus the condition of separation of $\mathscr{B}$ assures that every set of representatives $\left\{b_{i_{j}} \in H \cap B_{i_{j}} \mid 1 \leq j \leq d+1\right\}$ will have the same order type, and we can talk about the order type of $\left\{H \cap B_{i_{1}}, \ldots, H \cap B_{i_{d+1}}\right\}$ itself.

In $\S 2$ we discuss the notion of a minimal Radon partition, which turns out to be crucial to the proof, and prove a key property which may be thought of as a "Radon exchange principle." This is then used in $\S 3$ to prove Theorem 1. We conclude with a few remarks.

We express our appreciation to a referee for suggesting a shorter proof of Lemma 2 and for some salient comments about oriented matroids.

\section{Minimal Radon PaRtitions}

In order to generalize Hadwiger's theorem we make use of the notion of a minimal Radon partition, and we begin with a few elementary facts about these. For the purposes of this paper, a minimal Radon partition (MRP) in $\mathbf{A}^{d}$ will mean a set of $d+2$ points of $\mathbf{A}^{d}$ in general position, together with a decomposition $S=T \cup V$ into two subsets with the property that

$$
\operatorname{conv}(T) \cap \operatorname{conv}(U) \neq \varnothing .
$$

Why "minimal"? Because it is easily seen that a set of $d+1$ points in general position has no such decomposition (they form the vertices of a simplex, and any subset can be split from its complement by a suitable hyperplane); on the 
other hand, Radon's theorem (see for example [2]) asserts that for $d+2$ points such a decomposition always exists. Moreover, it is well known (and easy to see) that such a decomposition of $S$ is necessarily unique, hence we may speak of the MRP associated to a set $S$ of $d+2$ points in general position. If we identify $\mathbf{A}^{d}$ with $\mathbb{R}^{d}$, and write $S=\left\{x_{1}, \ldots, x_{d+2}\right\}$, then the MRP $S=T \cup V$ is a decomposition of $S$ into two sets, say $\left\{x_{1}, \ldots, x_{k}\right\}$ and $\left\{x_{k+1}, \ldots, x_{d+2}\right\}$, such that

$$
\sum_{i=1}^{k} a_{i} x_{i}=\sum_{i=k+1}^{d+2} b_{i} x_{i}
$$

for some choice of $a_{i}, b_{i}>0$ with $\sum a_{i}=1$ and $\sum b_{i}=1$. In other words, an MRP arises from writing $\sum a_{i} x_{i}=0$ with $\sum a_{i}=0$, and letting $T$ (resp. $V$ ) be the set of vertices having positive (resp. negative) coefficients.

Lemma 1. If $S=T \cup V$ and $S^{\prime}=T^{\prime} \cup V^{\prime}$ are each numbered configurations of points in general position in $\mathrm{A}^{d}$ having the same order type, with $T$ (resp. $V)$ corresponding to $T^{\prime}$ (resp. $\left.V^{\prime}\right)$ in the numbering, then the first is a Radon partition if and only if the second is.

Proof. The condition of $S=T \cup V$ not being a Radon partition entails the existence of a hyperplane spanned by $d$ of the points of $S$ which separates the remaining points of $T$ and $V$. (One sees this by rotating any separating hyperplane about increasingly constrained $d-2$-flats until it contacts $d$ of the points; the process is reversible.) Hence the condition of $S=T \cup V$ being a Radon partition is expressible purely in terms of the orientations of $d+1$-tuples of points of $S$, and this information is contained in the order type.

The following result can be viewed as an exchange principle for minimal Radon partitions. Surprisingly, it does not seem to appear in the literature surrounding Radon's theorem.

Lemma 2 (Radon exchange). If $S=T \cup V$ is an MRP in $\mathbf{A}^{d}$ and $x$ is any point of $\mathbf{A}^{d}$ in general position with respect to the points of $S$, then either

(1) $\exists t \in T$ such that $S \cup\{x\} \backslash\{t\}=(T \cup\{x\} \backslash\{t\}) \cup V$ is an $M R P$, or

(2) $\exists v \in V$ such that $S \cup\{x\} \backslash\{v\}=(T \cup\{x\}) \cup(V \backslash\{v\})$ is an MRP.

(Note the asymmetry in $T, V$. This says that if we wish to add a new point to $T$, we can do so either by removing a point of $T$ or, failing that, by removing a point of $V$.)

Proof. By hypothesis, there are constants $a_{i}, b_{i}>0$ such that

$$
\sum a_{i} t_{i}=\sum b_{i} v_{i}, \quad \sum a_{i}=\sum b_{i} .
$$

Since $x$ is affinely dependent on $S$, we may write

$$
x+\sum c_{i} t_{i}=\sum d_{i} v_{i}, \quad 1+\sum c_{i}=\sum d_{i}
$$


for some $c_{i}, d_{i}$ not necessarily positive. We wish to conclude from (1) and (2) that

$$
x+\sum e_{i} t_{i}=\sum f_{i} v_{i}, \quad 1+\sum e_{i}=\sum f_{i},
$$

with all $e_{i}, f_{i} \geq 0$ and some $e_{i}$ or $f_{i}=0$. But this follows immediately by subtracting $k$ times (1) from (2), where $k$ is the minimum of the ratios $c_{i} / a_{i}$, $d_{i} / b_{i}$.

(Note: It is not difficult to see that the point $t \in T$ or $v \in V$ which is replaced by $x$ has the following geometric description. If $C$ is the cell complex formed by all the hyperplanes joining sets of $d$ points selected from $S$, then $x$ lies in the interior of some cell $\Delta$ of $C$. If $\Delta$ is a bounded cell, precisely one vertex $t$ of $\Delta$ will belong to $T$; this is the point which $x$ replaces. If $\Delta$ is an unbounded cell, then either $x$ is adjacent to a (unique) vertex $t \in T$ of $\Delta$, in which case this is again the point which $x$ replaces, or else the antipodal cell $\bar{\Delta}$ of $\Delta$ will have precisely one vertex $v$ belonging to $V$; in the latter case it is $v$ which $x$ replaces.)

Lemma 3. If $K$ is a hyperplane in $\mathbf{A}^{d}$ and $S=T \cup V$ is an MRP in $K$, then any hyperplane $H$ which weakly separates $T$ from $V$ must be $K$ itself.

Proof. If the two closed halfspaces of $\mathbf{A}^{d}$ determined by $H$ contain $T$ and $V$ respectively, then the same is true of the two closed halfspaces of $K$ determined by $H \cap K$ (if $H$ and $K$ are distinct hyperplanes); but this is impossible since $S=T \cup V$ is an MRP in $K$.

Lemma 4. Given a set $S$ of $d+2$ points in general position in $\mathbf{A}^{d}$ partitioned into three sets, $S=T^{\prime} \cup V^{\prime} \cup W$, suppose no hyperplane through the points of $W$ separates $T^{\prime}$ from $V^{\prime}$. Then $\exists T \supset T^{\prime}$ and $V \supset V^{\prime}$ with $T, V \subset S$ such that $S=T \cup V$ is an MRP.

Proof. Let $A=\operatorname{conv}\left(T^{\prime} \cup W\right)$ and $B=\operatorname{conv}\left(V^{\prime} \cup W\right)$. Then $\operatorname{relint}(A) \cap$ $\operatorname{relint}(B) \neq \varnothing$, since otherwise $A$ and $B$ would be weakly separated by a hyperplane which would necessarily pass through the points of $W$, contrary to hypothesis. This implies that

$$
\sum a_{i} t_{i}^{\prime}+\sum b_{i} w_{i}=\sum c_{i} v_{i}^{\prime}+\sum d_{i} w_{i}, \quad \sum a_{i}+\sum b_{i}=\sum c_{i}+\sum d_{i},
$$

for some positive coefficients $a_{i}, b_{i}, c_{i}, d_{i}$, and-transposing the terms involving the $w_{i}$ so that each $w_{i}$ occurs only once-we get a relation of the desired form. (Notice that no $w_{i}$ cancels out, since then we would have a Radon partition of fewer than $d+2$ points, which is impossible.)

\section{Proof of Theorem 1}

We begin as in [5]. For each $i=1, \ldots, n$, fix a point $c_{i} \in B_{i}$. For $t \in[0,1]$, let $B_{i}(t)$ be the contraction of $B_{i}$ by a factor of $t$ about $c_{i}$; i.e.,

$$
B_{i}(t)=\left\{c_{i}+t\left(b-c_{i}\right) \mid b \in B_{i}\right\} .
$$


If $t_{1}<t_{2}$ and the hypothesis holds for $B_{1}\left(t_{1}\right), \ldots, B_{n}\left(t_{1}\right)$, then clearly it holds for $B_{1}\left(t_{2}\right), \ldots, B_{n}\left(t_{2}\right)$ as well. Hence, letting

$$
\tau=\inf \left\{t \mid \text { the hypothesis holds for } B_{1}(t), \ldots, B_{n}(t)\right\},
$$

we see that the hypothesis holds for $t>\tau$ and fails for $t<\tau$.

In fact, it holds for $t=\tau$ as well, by the following argument. Fix $i_{1}, \ldots, i_{d+1}$, let $t_{1}, t_{2}, \ldots$ be a decreasing sequence converging to $\tau$, and-for each $i \in$ $\left\{i_{1}, \ldots, i_{d+1}\right\}, j \in \mathbf{Z}$-let $y_{i}^{j} \in H_{j} \cap B_{i}\left(t_{j}\right)$ for a suitable oriented hyperplane $H_{j}$ with positive unit normal vector $v_{i}$. Replacing $t_{1}, t_{2}, \ldots$ by a subsequence, if necessary, we may assume, by compactness, that $y_{i}^{1}, y_{i}^{2}, \cdots \rightarrow y_{i} \in B_{i}$ for each $i \in\left\{i_{1}, \ldots, i_{d+1}\right\}$, and $v_{i} \rightarrow v$. Hence-by continuity-the oriented hyperplane $H$ with positive unit normal vector $v$ passing through $y_{i_{1}}$, say, contains all of $y_{i_{1}}, \ldots, y_{i_{d+1}}$, and their order type is weakly consistent with that of $\mathscr{C}$ (i.e., consistent except for possible degeneracies). However, no degeneracies can occur by the assumption that the original family $B_{1}, \ldots, B_{n}$ is separated, and so the hypothesis holds for $t=\tau$.

If $\tau=0$, the theorem is trivial since it is then immediate that all of the points $c_{1}, \ldots, c_{n}$ belong to a single hyperplane. Hence we may assume that the hypothesis is satisfied for the original convex sets $B_{i}$ but not for any proper contractions $B_{i}(t), t<1$. It follows that for some set of $d+1$ sets among $B_{1}, \ldots, B_{n}$, say for $B_{1}, \ldots, B_{d+1}$, there is an oriented hyperplane $H$ which meets them consistently with the order type of $\mathscr{C}$, but not for any proper contraction $B_{1}(t), \ldots, B_{d+1}(t), t<1$. We claim $H$ meets all of $B_{1}, \ldots, B_{n}$.

First notice that $H$ must be a supporting hyperplane to each of $B_{1}, \ldots, B_{d+1}$, i.e., that $H \cap B_{i} \neq \varnothing$, and $B_{i}$ is contained in one of the closed halfspaces determined by $H$. This is seen as follows. Suppose $H$ splits $B_{d+1}$. For each $i$, $1 \leq i \leq d$, we have both $B_{i} \cap H^{+} \neq \varnothing$ and $B_{i} \cap H^{-} \neq \varnothing$, where, as usual, $H^{+}$and $H^{-}$are the two closed halfspaces determined by $H$. Suppose first that no $B_{i}$ lies entirely in $H$, so that (say) $B_{1}, \ldots, B_{k}$ meet $\operatorname{int}\left(H^{+}\right)$and $B_{k+1}, \ldots, B_{d}$ meet $\operatorname{int}\left(H^{-}\right)$. Then letting $b_{i}$ be an arbitrary point of $B_{i} \cap H$ for each $i$, we see that

$$
\left\{b_{1}, \ldots, b_{d}\right\}=\left\{b_{1}, \ldots, b_{k}\right\} \cup\left\{b_{k+1}, \ldots, b_{d}\right\}
$$

cannot form a Radon partition in $H$, since (by the separation condition) these points are in general position in a $d$-1-plane, and there are only $d$ of them. Thus some $d$-2-flat $F \subset H$ separates them, and we can rotate $H$ around $F$ slightly, to a new hyperplane $H^{\prime}$, which now splits each of $B_{1}, \ldots, B_{d}$ and in addition continues to split $B_{d+1}$. (Figure 1 illustrates the case $d=2$.) Hence we can contract $B_{1}, \ldots, B_{d}$ further about $c_{1}, \ldots, c_{d}$, which is impossible.

Now suppose, more generally, that $B_{1}, \ldots, B_{j}$ are actually contained in $H$, and that $B_{j+1}, \ldots, B_{k}$ meet $\operatorname{int}\left(H^{+}\right)$, while $B_{k+1}, \ldots, B_{d}$ meet $\operatorname{int}\left(H^{-}\right)$. In this case, we let $b_{i}$ be an arbitrary point of $B_{i} \cap H$ only for $i=j+1, \ldots, d$, and choose $F \subset H$ not only separating $\left\{b_{j+1}, \ldots, b_{k}\right\}$ from $\left\{b_{k+1}, \ldots, b_{d}\right\}$, 


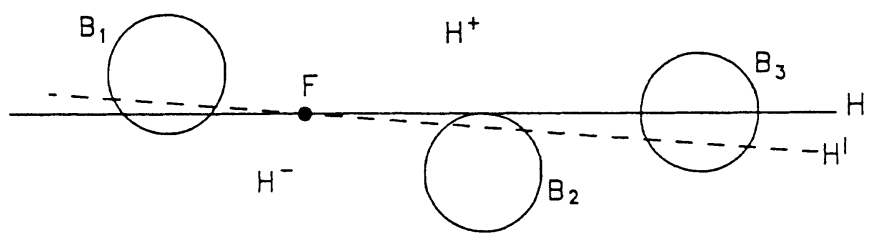

FIGURE 1

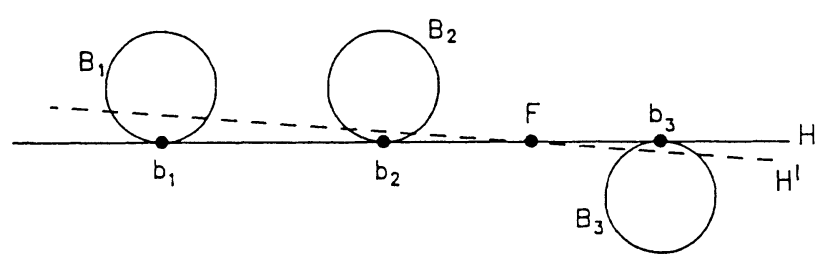

FIGURE 2

but also passing through the centers of contraction $c_{1}, \ldots, c_{j}$ (this can be done since $c_{1}, \ldots, c_{j}, b_{j+1}, \ldots, b_{d}$ are the vertices of a simplex in $H$ ). This guarantees that-after we rotate $H$ about $F$ as above-the new hyperplane will not only split $B_{j+1}, \ldots, B_{d+1}$, but pass through $c_{1}, \ldots, c_{j}$ as well. Hence, as above, we can contract $B_{1}, \ldots, B_{d+1}$ further about $c_{1}, \ldots, c_{d+1}$, which is impossible.

Since $H$ supports $B_{1}, \ldots, B_{d+1}$, we have $B_{i} \subset H^{+}$or $B_{i} \subset H^{-}$(or both, i.e. $\left.B_{i} \subset H\right)$ for each $i=1, \ldots, d+1$. Suppose first that none of $B_{1}, \ldots, B_{d+1}$ is contained in $H$, and that, without loss of generality, say $B_{1}, \ldots, B_{k} \subset H^{+}$ and $B_{k+1}, \ldots, B_{d+1} \subset H^{-}$, and-for each $i=1, \ldots, d+1$-let $b_{i} \in B_{i} \cap H$. In this case, we claim that

$$
\left\{b_{1}, \ldots, b_{d+1}\right\}=\left\{b_{1}, \ldots, b_{k}\right\} \cup\left\{b_{k+1}, \ldots, b_{d+1}\right\}
$$

is an MRP in $H$. Otherwise, as above, there would be a $d$-2-flat $F \subset H$ separating the first set from the second, and-by rotating $H$ slightly about $F$ in the proper direction-we could find a new hyperplane $H^{\prime}$ splitting each of $B_{1}, \ldots, B_{d+1}$ and still meeting them in the same order type, so that $H^{\prime}$ would still meet $B_{1}(t), \ldots, B_{d+1}(t)$ correctly for some $t<1$, which is impossible. (See Figure 2.)

It may happen, more generally, that not every one of $B_{1}, \ldots, B_{d+1}$ sticks out of $H$. Say $B_{1}, \ldots, B_{j} \subset H, B_{j}, \ldots, B_{k} \subset H^{+}$(but not $H^{-}$), and $B_{k+1}, \ldots, B_{d+1} \subset H^{-}$(but not $H^{+}$). Let $b_{i} \in B_{i} \cap H$ for $i=j+1, \ldots, d+1$. Then no $d$-2-flat $F$ through $c_{1}, \ldots, c_{j}$ can separate $\left\{b_{j+1}, \ldots, b_{k}\right\}$ from $\left\{b_{k+1}, \ldots, b_{d+1}\right\}$, since otherwise we could again rotate $H$ about $F$ slightly to arrive at a new hyperplane $H^{\prime}$ splitting each of $B_{j+1}, \ldots, B_{d+1}$ and still meeting them correctly, and at the same time passing through $c_{1}, \ldots, c_{j}$, so that $H^{\prime}$ 
would still meet $B_{1}(t), \ldots, B_{d+1}(t)$ correctly for some $t<1$, which is again impossible. Hence, by Lemma 4 , the points $c_{1}, \ldots, c_{j}$ may be partitioned into two sets, say $\left\{c_{1}, \ldots, c_{m}\right\}$ and $\left\{c_{m+1}, \ldots, c_{j}\right\}$, such that

$$
\begin{aligned}
& \left\{c_{1}, \ldots, c_{j}, b_{j+1}, \ldots, b_{d+1}\right\} \\
& \quad=\left\{c_{1}, \ldots, c_{m}, b_{j+1}, \ldots, b_{k}\right\} \cup\left\{c_{m+1}, \ldots, c_{j}, b_{k+1}, \ldots, b_{d+1}\right\}
\end{aligned}
$$

is an MRP in $H$.

Now let $p$ be any index $>d+1$. We will show that $H$ meets $B_{p}$. Suppose $B_{P} \subset H^{+}$. By Lemma 1, since the property of a union of two sets being a Radon partition is an invariant of the order type,

$$
\left\{x_{1}, \ldots, x_{d+1}\right\}=\left\{x_{1}, \ldots, x_{k}\right\} \cup\left\{x_{k+1}, \ldots, x_{d+1}\right\}
$$

is a Radon partition. Let us apply Lemma 2 to this MRP and the point $x_{p}$. Then, for some $j=1, \ldots, d+1$, either

$$
\left\{x_{1}, \ldots, \widehat{x}_{j}, \ldots, x_{d+1}, x_{p}\right\}=\left\{x_{1}, \ldots, \widehat{x}_{j}, \ldots, x_{k}, x_{p}\right\} \cup\left\{x_{k+1}, \ldots, x_{d+1}\right\}
$$

or

$$
\left\{x_{1}, \ldots, \widehat{x}_{j}, \ldots, x_{d+1}, x_{p}\right\}=\left\{x_{1}, \ldots, x_{k}, x_{p}\right\} \cup\left\{x_{k+1}, \ldots, \widehat{x}_{j}, \ldots, x_{d+1}\right\}
$$

is an MRP (depending on whether $j \leq k$ or $j \geq k+1$ ). By hypothesis, there is a hyperplane $K$ which meets $B_{1}, \ldots, \widehat{B}_{j}, \ldots, B_{d+1}, B_{p}$, and does so consistently with $\mathscr{C}$. If we let $b_{i}^{\prime} \in K \cap B_{i}$ for each $i=1, \ldots, \hat{j}, \ldots, d+1, p$, the points $b_{i}^{\prime}$ form an MRP in $K$, and moreover

$$
b_{i}^{\prime} \in H^{+} \text {for } i=1, \ldots, k \text { and } i=p,
$$

and

$$
b_{i}^{\prime} \in H^{-} \text {for } i=k+1, \ldots, d+1 .
$$

We can therefore invoke Lemma 3 and conclude that $H=K$, i.e., that $H$ meets $B_{i}$ as asserted.

\section{ReMARKS}

1. Hadwiger's theorem holds not only for a finite family of compact convex sets in the plane, but for any countable family $\mathscr{B}=\left\{B_{1}, B_{2}, \ldots\right\}$. The same simple compactness argument which Hadwiger uses in [5] to extend the result from finite to countable families works also in our case (we do not reproduce the details). The result is

Corollary. If $\mathscr{B}=\left\{B_{1}, B_{2}, \ldots\right\}$ is a countable separated family of compact convex sets in $\mathrm{A}^{d-1}$, then the members of $\mathscr{B}$ have a common transversal hyperplane if and only if there is a configuration of points $\mathscr{C}=\left\{x_{1}, x_{2}, \ldots\right\}$ in 


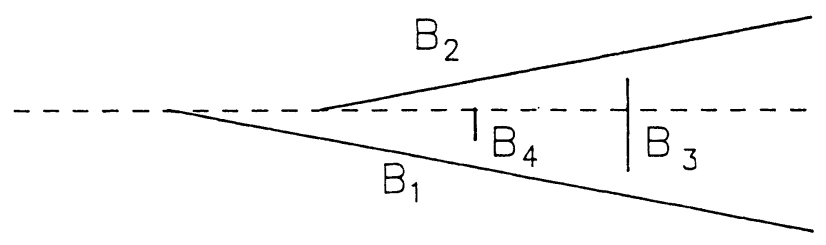

FIGURE 3

$\mathbf{A}^{d-1}$ such that for any set of $d+1$ indices $i_{1}, \ldots, i_{d+1} \in \mathbb{N}$ there is an oriented hyperplane which meets $B_{i_{1}}, \ldots, B_{i_{d+1}}$ consistently with the order type of $\mathscr{C}$.

2. We have no examples indicating that the separation condition in Theorem 1 is necessary for the result to hold, even in dimension 2; it would therefore be interesting to know to what extent, if any, it can be weakened. [Added in proof: Rephael Wenger has recently shown that separation can be removed completely in dimension 2 (R. Wenger, Hadwiger's theorem for intersecting convex sets, Discrete Comput. Geom. (to appear)); the question remains open, however, in higher dimensions.] It is surprising to note, however, that the condition in Theorem 1 does not imply the existence of a common transversal meeting the members of $\mathscr{B}$ in the same order type as $\mathscr{C}$, even though a casual reading of the statement would suggest that that is the case. Indeed, an example of G. Kertész [6] shows that even for $d=2$ this conclusion would be false; see Figure 3 , in which the sets $B_{1}, B_{2}, B_{3}, B_{4}$ have common transversals meeting every three in the corresponding order, but not all four.

3. Lemma 2 can also be proved for oriented matroids (see [1]), as Las Vergnas has pointed out. More generally, in fact, Theorem 1 still holds if we replace the configuration $\mathscr{C}$ by a simple oriented matroid; the proof is virtually unchanged in that case.

4. In view of Theorem 1, a natural question to investigate would now be: Are there order and separation conditions for a family of compact convex sets in $d$-space to have a transversal $k$-flat?

\section{REFERENCES}

1. R. Bland and M. Las Vergnas, Orientability of matroids, J. Combin. Theory Ser. B 24 (1978), 94-123.

2. L. Danzer, B. Grünbaum, and V. Klee, Helly's theorem and its relatives, Convexity, Proc. Symp. Pure Math., Vol. 7, Amer. Math. Soc., Providence, R. I., 1963, pp. 101-180.

3. B. Grünbaum, On a theorem of L. A. Santaló, Pacific J. Math. 5 (1955), 351-359.

4. J. E. Goodman and R. Pollack, Multidimensional sorting, SIAM J. Comput. 12 (1983), 484507.

5. H. Hadwiger, Über Eibereiche mit gemeinsamer Treffgeraden, Portugal. Math. 16 (1957), 2329.

6. G. Kertész, personal communication.

7. V. Klee, Common secants for plane convex sets, Proc. Amer. Math. Soc. 5 (1954), 639-641. 
8. F. A. Valentine, The dual cone and Helly type theorems, Convexity, Proc. Symp. Pure Math., Vol. 7, Amer. Math. Soc., Providence, R. I., 1963, pp. 473-493.

Department of Mathematics, City College, City University of New York, New York, NY 10031

Courant Institute of Mathematical Sciences, New York University, New York, NY 10012 